\title{
FEVER OF UNDETERMINED ORIGIN IN LUPUS AND LYMPHOMA: AN ASSOCIATION THAT SHOULD BE INVESTIGATED
}

Viviane Ferreira de Vasconcelos ${ }^{1, *}$, Laís Cristina Ferreira de Vasconcelos ${ }^{2}$, Daniela Aliny Tavares ${ }^{1}$, Luís Henrique Sarmento Tenório ${ }^{3}$, Karine Ferreira de Vasconcelos ${ }^{4}$, Izabel Crystine Pereira Barbosa ${ }^{5}$, Carolina Luísa Cardoso Militão ${ }^{6}$

1. Universidade Federal de Pernambuco, Recife (PE), Brazil. 2.Universidade Católica de Pernambuco, Recife (PE), Brazil. 3.Hospital Militar de Área de Recife, Recife (PE), Brazil. 4.Hospital da Restauração, Recife (PE), Brazil. 5.Hospital Central da Aeronáutica, Brazil. 6.Multihemo, Recife (PE), Brazil.

*Corresponding author:vivi_ferreiravasc@hotmail.com

\section{BACKGROUND}

The investigation of fever of unknown origin (FUO) is a major challenge in medicine, and, among autoimmune diseases, systemic lupus erythematosus (SLE) may be a possible hypothesis. Patients with SLE may present with fever due to disease activity, normally in these cases there will be consumption of complement and elevated SLEDAI, but infectious causes and even malignancies must be considered in the differential diagnosis.

\section{CASE REPORT}

A woman, 58 years old, with SLE for 6 years due to malar rash, photosensitivity, lymphopenia, leukopenia, thrombocytopenia, inflammatory arthralgia, complement consumption and positive FAN 1/1280 thick speckled nuclear. She was in control of disease activity using azathioprine $2 \mathrm{mg} / \mathrm{kg} /$ day and prednisone $2.5 \mathrm{mg} /$ day. In December 2019, she started a night fever (up to $40{ }^{\circ} \mathrm{C}$ ) with sweating, nausea, loss of $2 \mathrm{~kg}$ and abdominal pain. Chest computed tomography (CT) and abdominal USG on 12/16/19 showed only a $0.6 \times 0.4 \mathrm{~cm}$ noncalcified nodule in the lower lobe of the right lung. Laboratory tests with leukopenia (which was already the patient's standard), anemia with direct coombs negative and increased inflammatory tests, but without complement consumption and negative anti-DNA. When increasing the dose of prednisone to $20 \mathrm{mg}$, she showed clinical improvement, remaining afebrile, but with a dose reduction returned to having fever, being chosen hospitalization for investigation. Negative urine, feces and blood cultures, HIV serology, syphilis, Epstein-Barr (IgM), leishmaniasis, toxoplasmosis (IgM) and herpes (IgM) negative. In a new chest and abdomen CT about 15 days apart from the first, we observed bilateral and axillary lymph node enlargement bilaterally in mediastinal, paraortic, paratracheal and subcarinal, peritoneal and retroperitoneal chains, the largest measuring $3.0 \times 2.3 \mathrm{~cm}$. Bone marrow biopsy with malignant lymphoproliferative activity, PET-CT with metastases in the pelvis and femurs was performed. Anatomopathological study confirmed classical Hodgkin's lymphoma. The patient signed an informed consent form authorizing the case report.

\section{CONCLUSION}

The association of SLE with some malignancies, including lymphoma, is already known. Dysregulation of the immune system that occurs in lupus and its inflammatory process are implicated in this pathogenesis. Distinguishing the causes of fever in a patient with SLE is a difficult task, as there is always the question of whether the fever is due to disease activity or another process; however, this association should always be remembered, especially in patients without evidence of SLE activity and lymphadenomegaly because early diagnosis and immediate treatment initiation are decisive for the prognosis. 